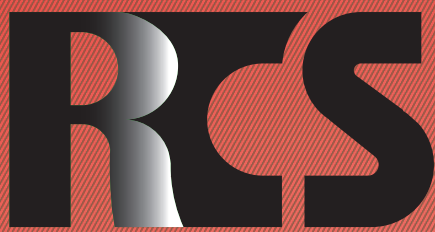

Depósito legal ppi $201502 Z U 4662$

Esta publicación científica en formato digital es continuidad de la revista impresa Depósito Legal: pp $197402 Z$ Z789

- ISSN: 1315-9518 • ISSN-E: 2477-9431

Revista de Ciencias Sociales

Universidad del Zulia. Revista de la Facultad de Ciencias Económicas y Sociales Vol. XXVII,

No. 4,2021

Esta publicación científica en formato digital es continuidad de la revista impresa Depósito Legal: pp $197402 Z 1789$ ISSN: $1315-9518$ 


\title{
Crisis empresarial, factores que influyen y alteran la gestión de las empresas en Colombia
}

\author{
Olis Barreto, Irma María* \\ Reyes, Giovanni E.** \\ Martin-Fiorino, Víctor ${ }^{* * *}$ \\ Villalobos-Antúnez, José Vicente ${ }^{* * * *}$
}

\section{Resumen}

Este artículo indaga los factores que intervienen en la crisis empresarial, los modelos y tipos de crisis que afectan y limitan las intervenciones de las empresas colombianas en su entorno. El tipo de metodología es la de un estudio exploratorio cualitativo interpretativo acerca de la crisis constituida por un choque simultáneo de oferta y demanda que afectó a todos los sectores sociales, en particular a los más vulnerables. Se realizó un estudio exploratorio a manera de fase inicial por razones de recursos, en particular el recurso tiempo. El estudio exploró las causas que llevaron a la crisis, tomando como muestra a ocho empresas colombianas, su proceso de resurgimiento y estrategias para su continuidad. Fueron realizadas entrevistas a cuadros medios y gerentes de empresas de servicios de logística y de educación, y 155 encuestas a empresarios y personal administrativo. Los hallazgos sostienen que existen factores como la gestión financiera, eficiencia en procesos y gestión integral que marcan un horizonte con escenarios comprometedores para el futuro empresarial. Se concluye, que es significativo para la empresa captar las señales de alerta, determinar su procedencia y conocer sus problemáticas para permitir el pronóstico de una crisis empresarial, evitando llegar al punto de no retorno.

Palabras clave: Crisis empresarial; entorno empresarial; eficiencia en procesos; gestión integral; gestión financiera.

* Estudiante de Doctorado en Ciencias de la Educación. Magister en Desarrollo Educativo y Social. Posgrado Gerencia en Gestión Humana y Desarrollo Organizacional. Profesora de carrera de la Escuela de Administración en la Universidad del Rosario, Colombia. E-mail: irma.olis@urosario.edu. co (iD) ORCID: https://orcid.org/0000-0002-5440-8576

** PhD. Doctorado en Economía para el Desarrollo / Relaciones Internacionales, de la Universidad de Pittsburgh. Profesor Titular y Director de la Maestría en Dirección en la Universidad del Rosario, Bogotá, Colombia. E-mail: giovanni.reyes@urosario.edu.co (iD ORCID: https://orcid.org/0000-0001$\underline{6172-6890}$

**** PhD. Doctorado en Filosofía de la Universidad de Lovaina, Bélgica. Docente e Investigador en la Universidad El Bosque, Bogotá, Colombia. Miembro del Grupo de Investigación Gintecpro. E-mail: vmartinf@unbosque.edu.co (iD) ORCID: https://orcid.org/0000-0003-4057-7974

**** PhD. en Derecho Docente en la Universidad de la Costa, Barranquilla, Colombia; Universidad Católica de Temuco, Chile; y Universidad del Zulia, Venezuela. E-mail: jvillalo4@cuc.edu.co (iD ORCID: https://orcid.org/0000-0002-3406-5000

Recibido: 2021-06-19

Aceptado: 2021-09-05 


\title{
Business crisis, factors that influence and alter the management of companies in Colombia
}

\begin{abstract}
This article investigates the factors that intervene in the business crisis, the models and types of crisis that affect and limit the interventionsof Colombian companies in their environment. The type of methodology is that of an interpretive qualitative exploratory study about the crisis constituted by a simultaneous supply and demand shock that affected all social sectors, particularly the most vulnerable. An exploratory study was carried out as an initial phase for reasons of resources, particularly time resources. The study explored the causes that led to the crisis, taking as a sample eight Colombian companies, their resurgence process and strategies for their continuity. Interviews were conducted with middle managers and managers of education and logistics services companies, and 155 surveys of businessmen and administrative personnel. The findings maintain that there are factors such as financial management, process efficiency and comprehensive management that mark a horizon with compromising scenarios for the future of the business. It is concluded that it is significant for the company to capture the warning signs, determine their origin and know their problems to allow the forecast of a business crisis, avoiding reaching the point of no return.
\end{abstract}

Keywords Business crisis; business environment; process efficiency; Integral management; financial management.

\section{Introducción}

En el pensamiento contemporáneo, el concepto de empresa se ha ido resignificando desde diversas perspectivas y en entornos complejos. En tal sentido, cabe considerar que la empresa representa un sistema que interactúa con diferentes estándares y recursos provenientes de su entorno. Estos juegan un rol fundamental en los procesos internos y externos de la empresa, pero también en la generación de cambios. Las presiones que presenta el entorno la afectan positiva o negativamente, por ello la importancia de su capacidad de búsqueda de la eficiencia y de sus procedimientos en todos los escenarios.

Desde el pensamiento empresarial de la transición entre el siglo XX y el siglo XXI, la empresa es considerada como un subsistema, inserto en su medio y orientada hacia ciertas metas (Kast y Rosenzweig, 1972). A partir de esas bases, replanteadas en los actuales contextos de Colombia, es preciso que la empresa explore los cambios que se producen en su entorno y preste atención a ellos, dado que se encuentra expuesta a múltiples factores que pueden generarle dificultades, y por lo cual, debe tomar acciones que le permitan gestionar procesos y minimizar sus riesgos.

Sin embargo, aunque pareciera que los cambios que suceden en un momento de crisis empresarial dan lugar a dinámicas internas y externas, es posible que la empresa también se encuentre expuesta diariamente a varios factores de riesgo, particularmente por las dificultades económicas y financieras en los entornos hostiles en los que viven hoy en día incluyendo la realidad de Colombia donde hubo una contracción del 7\% de la producción y la pobreza llego a $42 \%$. También, es viable que tales situaciones puedan traer consigo inevitables consecuencias que alcanzan a generarle conflictos o dejarla vulnerable en relación con los escenarios del país en el futuro cercano.

Es en esos momentos de crisis, cuando la 
empresa se prepara para apropiarse y adaptarse a las diversificaciones del entorno, tal como se presenta en las condiciones de Colombia, de organizarse y regularse, pudiendo al mismo tiempo tener un relativo margen de albedrío para tomar decisiones, así como gestionar estrategias que le permitan desarrollarse y hacer frente al pronóstico de una crisis (que en Colombia país parece asociada al choque simultáneo de la oferta y la demanda, con el agravamiento de la situación de la población más vulnerable).

Esto implica conocer la realidad empresarial, implementar procesos que han de ser actividades sistemáticas y planificadas, $\mathrm{y}$ que tienen como finalidad proporcionar información para la toma de decisiones con vista a mejorar o transformar la realidad, facilitando los medios para llevarla a cabo (Pérez, 2002). Todo lo anterior lleva a la necesidad de hacer una caracterización de los principales desafíos de las empresas al enfrentar la actual crisis. Por lo cual, el objetivo del presente estudio consiste en indagar los factores que intervienen en la crisis empresarial, los modelos, así como tipos de crisis que afectan y limitan las intervenciones de las empresas colombianas en su entorno.

\section{Fundamentación teórica}

\subsection{Entorno empresarial}

El concepto de entorno hace referencia a todos aquellos factores que generan un impacto y desestabilizan la organización, exigiéndole que tenga miradas dinamizadoras de competitividad en el caos y las complejidades (Maldonado, 2009. Las acciones del entorno sobre la empresa, son de doble sentido, producen adaptación a su medio, pero también la modificación como consecuencia de la influencia que ejerce la empresa sobre el mismo. Es decir que, se entiende el entorno como todo aquello que es ajeno a la empresa, es decir, que viene dado por un conjunto de actores y fuerzas externas que, siendo parcial o totalmente incontroladas, son susceptibles de afectar la empresa.

Existen diferentes tipos de entorno: Está el global, en los cuales la empresa tiene poca o nada influencia directa, compuesto por diferentes elementos, que más o menos se relacionan entre sí: Político, económico, jurídico, tecnológico, internacional, psicosociológico y climáticos. Y el entorno específico, el cual tiene que ver con el sector en el que se encuentran los intereses de la empresa, concierne claramente con su actividad. Se basa en las fuerzas competitivas de Porter (2001), porque observa el ambiente más cercano a la empresa, y aporta importantes criterios para las tácticas de posicionamiento en el ámbito nacional e internacional. Está compuesto por proveedores, clientes y competidores.

A la hora de analizar el entorno empresarial, desde la teoría de los closter competitivo de Porter y para buscar su propio modelo de gestión empresarial en función de estas variables, al empresario le corresponde tener en cuenta factores como: Diferencias individuales entre empresas, los recursos con los que cuenta y el entorno en el que se desarrolla, dado que es cambiante en función de las empresas, las personas, los productos o servicios y las tareas que realiza, para así buscar su propio modelo de gestión empresarial en función de estas variables (Porter, 2007).

A partir de estas reflexiones, la empresa es susceptible de ser considerada un sistema abierto, porque puede realizar intercambios con su entorno, está en un permanente equilibrio dinámico, tiene la capacidad para realizar cambios o ajustes a su comportamiento, según el entorno y sus relaciones, y en efecto, no sería posible sin un proceso continuo de flujo de entradas, transformaciones y salidas. Usualmente, la empresa está adaptada a vivir en situaciones que se denominan niveles normales, sabe cómo actuar en algunos casos, y lo hace de manera efectiva. Pero, cada decisión que toma tiene consecuencias en un entorno cada vez más heterogéneo y en rápido desarrollo.

Por eso, cualquier situación imprevista trae repercusiones que la pueden llevar a la crisis; y una decisión o estrategia que 
Olis Barreto, Irma Maria; Reyes, Giovanni E.; Martin-Fiorino, Victor y

Villalobos-Antúnez, José Vicente

Crisis empresarial, factores que influyen y alteran la gestión de las empresas en Colombia

se tome en la organización, es importante deliberarla y evaluarla teniendo en cuenta el entorno, porque es quien fija la pauta para la toma de decisiones en la empresa, y lo hace sobre temas específicos que se relacionan con la identificación, evaluación, así como reacción ante las fuerzas externas, definición de las decisiones económicas y tecnológicas, la definición del complejo de leyes y relaciones del gobierno sobre la organización empresarial, y finalmente, la evaluación de la responsabilidad social de la empresa ante la comunidad (Castro, 2007; Bom-Camargo, 2021).

Sin duda, estas acciones colectivas, señalan la competitividad de la empresa, y probablemente su estabilidad. Pero también, son aspectos que desarrollan ventajas competitivas sostenibles que le permiten a la empresa garantizar un desempeño superior al de sus competidores y anticipar las tendencias globales si los valores nacionales se están propagando (Gran, 2005; Porter, 2007; Barney, 2010; Blanco-Ariza et al., 2020), y con ellas despliega sus propias capacidades para afrontar los riesgos del entorno, sobrevivir en algunos y en otros no (Sansores et al., 2020), identificar las oportunidades de desarrollo, generar acciones para gestionar internamente la administración de la empresa y lograr los objetivos.

Paralelamente, la empresa tiene la capacidad para adaptarse a los cambios del entorno, siendo esto una característica esencial, pero aquellas que no lo logran o que lo hacen tardíamente, normalmente están condenadas a la muerte empresarial, pues, como lo señalan Hannan y Freeman (1977), el entorno es el encargado de seleccionar a los individuos que subsisten en el medio.

Sobre el tema, son diversos los autores que argumentan acerca de los criterios en el tipo de factores que intervienen, así como afectan el entorno de la empresa, y coinciden en que existen características en el entorno que ciertamente van a condicionar las decisiones en la empresa (ver Cuadro 1).

\section{Cuadro 1}

\section{Tendencias del Entorno}

\begin{tabular}{ll}
\multicolumn{1}{c}{ Autores } & \multicolumn{1}{c}{ Tendencias } \\
\hline Levy y Hadar (2010) & La Web 2.0 dentro del contexto empresarial - TIC's \\
& $\begin{array}{l}\text { Las tendencias del entorno están orientadas a la } \\
\text { convergencia, tratando de integrar los variados } \\
\text { conocimientos de las diferentes ciencias y considerar la } \\
\text { empresa como un sistema abierto, en interrelación con el } \\
\text { entorno }\end{array}$ \\
Agulló (1999) & $\begin{array}{l}\text { Estable o dinámico - Simple o complejo - Integrado o } \\
\text { diverso - Hostil }\end{array}$ \\
\hline
\end{tabular}

Fuente: Elaboración propia, 2020.

Se evidencia, cómo los autores coinciden en abordar diversas tendencias del entorno. En algunos casos, estas características no consiguen ser controlables por la empresa, por ello, se ve en la necesidad de apropiarse de enfoques más estratégicos para inducir el incremento de la productividad y eficacia. Ante este panorama, es posible que la empresa no consiga actuar como un todo ante estos retos e incertidumbres y se encuentre en una posición desventajosa, lo que conlleva a realizar ajustes en sus estrategias, así como 
en su funcionamiento interno, en el que tenga en cuenta un entorno más abierto, o lo que involucra incrementos en términos de recursos y capacidad para responder a las exigencias dinámicas del entorno. Innegablemente, que gran parte de la evolución que ha tenido lugar en la dirección y organización de las empresas se han originado en los cambios del entorno (Agulló, 1999).

Finalmente, las medidas que se tomen consiguen generarle beneficios, implicaciones negativas, o situaciones de crisis. Es una encrucijada de cara a los retos de una economía en constante cambio, a las características del entorno, a las fortalezas y debilidades de la empresa para posicionarse, así como tener ventajas competitivas.

\subsection{Efectos de la crisis empresarial}

Sobre la crisis empresarial se ha discutido mucho, pero ¿qué es realmente? o ¿qué se conoce sobre ella? No existe un concepto preestablecido, pero si hay una serie de pautas que en esta sección se precisarán para describir el alcance o efecto de la crisis en la empresa.

\section{a. Análisis y trascendencia de la crisis}

La crisis es una palabra que proviene del griego krinein (cambio, transformación) y de esta palabra se deriva kritikós, que significa "crítico", "criticar", "discernir". La crisis aplicada al entorno empresarial, se refiere a un acontecimiento que puede ocasionar resultados negativos. Sin embargo, hay empresas que, cuando realizan un buen manejo de la misma, logran convertirla en una oportunidad de mejora. Es considerada una situación que crea inestabilidad en la empresa, son tan repentinas, vulneran a la empresa, o en algunos casos la expone a peligros.

Dado que krisis (krinein) en griego significa transición, coyuntura de cambio de una realidad sujeta a evolución, ello implica para la empresa un momento de toma de conciencia de su propia realidad en transformación, y al mismo tiempo con efecto transformador del entorno. Este significado se puede vincular a la percepción de una situación de oportunidad, así como también, al asociarse con el concepto de criterio (derivado de la misma raíz), se relaciona con el concepto de razonamiento adecuado, con lo que la crisis representa una situación que obliga a pensar, que genera un necesario proceso de reflexión y análisis. Subrayando un aspecto que con toda vigencia es aplicable al momento presente de las empresas, Tommasetti y Martin (2013) afirman que:

En los actuales escenarios de crisis, es necesario crear las condiciones para poder elegir el tipo de empresa que surgirá de ellos. No se trata de una expresión de deseos: se trata de elegir en sentido fuerte, potenciando la acción de redes de actores sociales (universidad, empresa, Estado, sociedad) capaces de alcanzar acuerdos y de ejercer una influencia real sobre la configuración de nuevas formas responsables hacer empresa. (p.104)

La crisis empresarial, manifiesta algo más serio que un simple problema y por lo general obstaculiza el flujo normal del negocio. Son eventos específicos que pueden romper el equilibrio de la organización, dependiendo del tamaño de la compañía, del número de empleados que ésta tenga, del producto o del servicio que preste al entorno. Teniendo en cuenta lo expuesto anteriormente, y debido al gran impacto que las crisis tienen en las organizaciones, es posible encontrar consideraciones o definiciones teóricas de diferentes autores que buscan explicar el alcance de la crisis, las cuales se muestran en el Cuadro 2. 


\section{Cuadro 2}

\section{Definición de crisis}

\begin{tabular}{ll}
\hline \multicolumn{1}{c}{ Autores } & \multicolumn{1}{c}{ Conceptos de crisis } \\
\hline $\begin{array}{l}\text { Antonacopoulou y } \\
\text { Sheaffer (2014)- }\end{array}$ & La crisis demanda prever para reducir sus efectos. \\
$\begin{array}{l}\text { McCray, Gonzalez y } \\
\text { Darling (2012) }\end{array}$ & $\begin{array}{l}\text { Son hechos externos a una empresa que no se refieren a deficiencias gerenciales o ausencia } \\
\text { de planificación, acciones conocidas como mala gestión. }\end{array}$ \\
Veil (1973) & $\begin{array}{l}\text { Son situaciones que llegan por sorpresa caracterizadas por tener efectos devastadores y por } \\
\text { tal razón se perciben dentro de la empresa como los últimos instantes antes de terminar por } \\
\text { completo su período de vida. }\end{array}$ \\
Deverell y Olsson (2010) & $\begin{array}{l}\text { La crisis se causa por eventos externos y representa un reto para el cambio. La crisis no } \\
\text { debe tomarse como inesperadas, a pesar de considerarse como difíciles de predecir. }\end{array}$ \\
Pearson y Clair (1998) & $\begin{array}{l}\text { Es un evento de alto impacto y baja probabilidad que pone en peligro la viabilidad de la } \\
\text { empresa y se caracteriza por su ambigüedad en la causa, efecto y los medios de resolución, } \\
\text { así como por el credo de que las decisiones deben tomarse rápidamente. }\end{array}$ \\
$\begin{array}{l}\text { Las crisis se considera como una "situación en que la reputación, prestigio y/o } \\
\text { supervivencia de una organización se ven fuertemente amenazadas por el acaecimiento de } \\
\text { un evento de distinta índole" }\end{array}$ \\
\hline
\end{tabular}

Fuente: Elaboración propia, 2020.

La crisis ha sido siempre determinante en cualquier circunstancia de la empresa. Por lo tanto, hay que tener presente que una crisis no debe ser siempre vista con negatividad, pues es necesario que la empresa pueda adaptarse a las condiciones del entorno para entregar lo mejor de sí mismas al ambiente que la rodea.

\section{b. La crisis según su categoría, clasificación y fases}

Así como son varias las definiciones de crisis, también lo son los tipos, categorías, clasificaciones y fases, y en cada una de ellas, algunos autores aportan un nuevo punto de vista para esta investigación, tal como se evidencia en el Cuadro 3, en el que se hace una comparación. 


\section{Cuadro 3}

Tipos de crisis según sus categorías, clasificación y fases

\begin{tabular}{lll}
\hline \multicolumn{1}{c}{ Categorías } & \multicolumn{1}{c}{ Clasificación } & \multicolumn{1}{c}{ Fases } \\
\hline Niveles de afectados & $\begin{array}{l}\text { Global; Internacional } \\
\text { Nacional, Regional y Local }\end{array}$ & $\begin{array}{l}\text { Nacimiento; desarrollo; } \\
\text { madurez y declive }\end{array}$ \\
$\begin{array}{l}\text { Posibilidades de intervención de } \\
\text { la organización }\end{array}$ & $\begin{array}{l}\text { Crisis evitables; Crisis no evitables; Crisis } \\
\text { accidentales u operativa }\end{array}$ & $\begin{array}{l}\text { Detección de señales; } \\
\text { Preparación y prevención; } \\
\text { Contención de daños; } \\
\text { Recuperación y aprendizaje }\end{array}$ \\
$\begin{array}{l}\text { Naturaleza y magnitud } \\
\text { Naturaleza de los } \\
\text { acontecimiento o lo que las } \\
\text { causan }\end{array}$ & $\begin{array}{l}\text { Objetivos; Técnicos/Políticos; Exógenos/ } \\
\text { Endógenos }\end{array}$ & $\begin{array}{l}\text { Preliminar; Aguda; Crónica; } \\
\text { Postraumática }\end{array}$ \\
\hline
\end{tabular}

Fuente: Elaboración propia, 2020, a partir de los aportes de Pearson y Mitroff (1993); Westphalen y Piñuel (1993); y, González (2008).

Posiblemente, los tipos de crisis citados anteriormente no son todos los que existan, pero pueden proporcionar una idea bastante completa de las crisis más habituales en las empresas. El que se hayan ubicado en una fila en particular, no quiere decir que siempre se presenten en el mismo lugar o entorno, y por ende es importante que la empresa esté alerta en todo momento.

\subsection{La perdurabilidad empresarial}

La perdurabilidad se encuentra asociada con conceptos como éxito, longevidad, supervivencia, hiperlongevidad y visión (Rivera, 2012; Rivera y Cardona, 2012). Por su parte, Toca (2011) sostiene que la perdurabilidad, "es una habilidad de los organismos para vivir o continuar viviendo por un período de tiempo más largo que otros" (p.6). Otro aspecto teórico que hace reflexionar sobre una empresa que perdura, es el citado por Collins y Porras (2006), cuando refiere que la empresa es visionaria, porque son instituciones que constituyen la flor y nata -las joyas de la corona-de su industria, admiradas por todos sus colegas y que tienen la larga tradición de haber ejercido una influencia significativa en el mundo que las rodea.

Como lo plantea Vélez (2005), la perdurabilidad empresarial "es el objetivo de la administración, es la filosofía de la eficiencia y la productividad [...] la perdurabilidad empresarial es el sentido de la administración $\mathrm{y}$ el alcance de su discurso en nuestros contextos" (p.89); y un esfuerzo por encontrar elementos que permitan a una empresa vivir muchos años, calidad de vida (Rivera, 2012). Para la Escuela de Administración de la Universidad del Rosario, la perdurabilidad es: Aquella que a través del tiempo presenta resultados financieros superiores. Adecua su manejo a la intensidad de las condiciones del entorno sectorial y las fuerzas del mercado. Se enfoca en espacios no explotados y hace un estudio detallado de sus competidores diseñando y ejecutando productivamente la cadena de valor. Es aquella que obtiene desempeños eficientes en su gestión por la coherencia en su acción, la identificación de su entorno sectorial y sus políticas de gobierno, evitando estados de morbidez que dificultan su crecimiento rentable y que puede llegar a estados tánaticos. Propicia la alineación de las 
Olis Barreto, Irma Maria; Reyes, Giovanni E.; Martin-Fiorino, Victor y

Villalobos-Antúnez, José Vicente

Crisis empresarial, factores que influyen y alteran la gestión de las empresas en Colombia

personas con la empresa, la construcción de conocimiento y la calidad en los procesos de interacción social. (Restrepo et al., 2009, p.57)

Este concepto surge después de realizar una encuesta tipo escala Likert, a 318 empresarios, para identificar las condiciones de perdurabilidad en las empresas colombianas. Fue un estudio realizado por la Escuela de Administración de la Universidad del Rosario, en la cual la información obtenida se procesó, y se realizó un análisis factorial, identificando trece componentes de perdurabilidad (Restrepo et al., 2009). Para el caso de este artículo, se analizaron tres componentes que se evidencian como factores que contribuyeron a la situación de crisis en ocho empresas colombianas objeto de estudio, las cuales se describen a continuación:

\section{a. Gestión integral}

La organización, es capaz de reproducirse y desarrollarse, posee una autonomía relativa, auto-regulada y autogestionada, una administración racional, capaz de adaptarse a los factores del entorno que la rodea (Koontz, Weihrich y Cannice, 2012). Posee fuerzas que motivan el crecimiento y el cambio, pero también, el mantenimiento de una armonía y equilibrio. La perturbación presenta una variabilidad, en su funcionamiento, así como en las dinámicas que surgen de ella misma. Dichos trastornos, pueden surgir por diferentes factores de su entorno general o específico, que de no ser atendidas pueden, eventualmente, encaminar a situaciones adversas o crisis empresariales.

La empresa, en continua interacción con su entorno, está expuesta a un ambiente con mucha información que está cambiando permanentemente; por lo cual, es preciso contar con una gestión que integre los procesos administrativos para la consecución de los objetivos (Márquez et al., 2021). Por lo que, la gestión integral le permite a la empresa vincular los inputs, con un mensaje claro y preciso, con el fin de optimizar, mejorar y ser eficiente en el funcionamiento de los sistemas $\mathrm{y}$ en la toma de decisiones.

Con la gestión integral se generan acciones normativas, operativas, financieras, administrativas, sociales, de planeación, seguimiento y control que se articulan e interrelacionan. El propósito, es detectar aquellos factores que intervienen en la empresa y que pudieran convertirse en crisis, o influenciar con efectos perjudiciales para la organización. Por ello, es conveniente hacer un análisis del entorno, conocer los potenciales conflictos, de manera que la empresa pueda disponer del tiempo necesario para evaluarlos y establecer estrategias.

No es fácil para la empresa determinar si se está ante una amenaza o una oportunidad, por ello para gestionar una crisis es significativo contar con los departamentos, áreas o unidades estratégicas, esencialmente, con el ser humano individual, así como las organizaciones con las que interactúa y su comunidad. Entonces, la gestión de la empresa estudia y analiza su entorno, sus comportamientos, para comprender las relaciones que surgen de allí. Según Blanchard, Dell'Ariccia y Mauro (2010), para no improvisar, la empresa debe considerar ocho principios en la crisis, los cuales deben estar presentes en el momento de gestionar la misma:

1. Compresivo: El gestor debe considerar $\mathrm{y}$ tener en cuenta todas las situaciones que generen problemáticas, fases y grupos de interés relacionadas con la crisis.

2. Preventiva: El gestor debe anticipar las crisis futuras y tomar acciones preventivas y medidas preparatorias para construir comunidades resistentes al desastre.

3. Manejar contingencias: El gestor debe utilizar la identificación de problemas, análisis de riesgo y análisis de impacto para la asignación de prioridades y recursos.

4. Integrada: El administrador asegura que la unión de esfuerzos sea a lo largo de todos los niveles de la gerencia y componentes de la comunidad.

5. Colaborativa: El gestor debe crear y sostener amplias, así como sinceras relaciones entre los individuos y organizaciones para 
promover la confianza, forjar una atmósfera de equipo, generar consenso, y facilitar la comunicación.

6. Coordinada: El gestor sincroniza las actividades de todos los grupos de interés relevantes para conseguir un propósito común.

7. Flexible: Debe sincronizar las actividades de todos los grupos de interés relevantes para conseguir un propósito común.

8. Profesional: El gestor valora una aproximación científica, basada en el conocimiento, con fundamentos en la educación, entrenamiento, experiencia, prácticas éticas y mejora continua.

Conviene realizar la gestión integral en la empresa, en la fundamentación y utilización de herramientas y técnicas, cada vez más actualizadas, para realizar el análisis de su desempeño en forma eficiente, con el fin de tomar decisiones acertadas; y con implicaciones, como lo plantean Jaques (2010); y Blanco-Ariza et al. (2020), para el desarrollo de la estructura organizacional. Siendo la gestión integral un elemento importante en la administración y en la prevención de la crisis.

En la perspectiva de este trabajo, la gestión de las empresas en relación con el entorno, tiene que verse como un instrumento de adaptabilidad de las organizaciones para enfrentar la turbulencia de la crisis en la pandemia en el entorno colombiano. Esta perspectiva ha mostrado las fortalezas $y$ debilidades de las empresas, en relación con los factores analizados

\section{b. Eficiencia en los procesos}

Es conveniente conocer lo que se entiende por eficiencia, por ello en el Diccionario de la Real Academia Española (2021), se considera como una virtud y facultad para lograr un efecto determinado. Para Martín, Hernangómez y Martín (2007), es la relación entre el nivel de objetivos alcanzados y el volumen de recursos utilizados. Afín con esta enunciación, se entiende como eficiencia el grado en que se cumplen los objetivos propuestos a un menor costo posible, y el no cumplimiento hace que resulten ineficientes.

Pero también lo es, evaluar la eficiencia en los procesos, necesario para establecer estándares adecuados, recursos empleados y una valoración de todas las dimensiones que pueden tener como objetivo la empresa. Así como pensar que los cambios tanto del entorno como los internos, alcanzan a ocasionar diferentes perjuicios a la organización, que eventualmente podrían llevarla a la crisis, o la motivan a un desarrollo y crecimiento permanente con miras a su sostenibilidad en un entorno cada vez más competitivo. Pero también, a gestionar un conjunto de actividades que se realizan con la finalidad de lograr un producto determinado, con capacidad para generar procesos de transformación de la realidad (Valero, 2012).

En general, la eficiencia en los procesos de las empresas que han pasado por crisis sufre una rápida transformación, desde cambios en las técnicas administrativas hasta en los equipos de trabajo. Pero también, conviene realizar una innovación organizacional, es decir, uso de tecnologías de información, y telecomunicaciones, a la reorganización de los intereses productivos, pero sobre todo a la innovación de sus procesos, y se resalta al entorno como factor de perdurabilidad empresarial, por ser una construcción humana; en ese sentido, para garantizar su perdurabilidad, debe ser capaz de asimilarse culturalmente como grupo de interés, lo que conlleva a darle gran importancia al factor entorno como incidente (Porter, 2007).

Acerca de este punto, el análisis cumplido en el presente trabajo muestra que la eficacia de los procesos tuvo mayores desafíos de adaptabilidad, en especial porque se registró la tendencia a interrumpir las cadenas de suministro.

\section{c. Gestión financiera}

Es una de las actividades significativas $\mathrm{y}$ trascendentales para el buen funcionamiento de la organización, puesto que una disposición financiera equivocada puede llevar a la crisis a 
Olis Barreto, Irma Maria; Reyes, Giovanni E.; Martin-Fiorino, Victor y

Villalobos-Antúnez, José Vicente

Crisis empresarial, factores que influyen y alteran la gestión de las empresas en Colombia

la empresa o la interrupción de sus actividades. Por ello, las empresas que resisten algunos entornos difíciles, le corresponde realizar medidas que le permitan ser más competitiva y eficiente desde la perspectiva económica, así como financiera.

En ese contexto, los objetivos de una gestión financiera se encaminan a analizar las tendencias de las variables financieras involucradas en las operaciones de la empresa; evalúa la situación económica y financiera para determinar el nivel de cumplimiento de los objetivos prestablecidos; verifica la coherencia de la información contable con la realidad de la empresa; identifica los problemas existentes, aplica los correctivos pertinentes, y orienta a la gerencia hacia una planificación financiera eficiente y efectiva (Nava, 2009; Báez y Puentes, 2018; Martínez et al., 2020). Todo ello, encaminado hacia el logro de los objetivos planteados por la organización, porque las exigencias del entorno hacen indispensable que las empresas estén preparadas para gestionar sus recursos financieros, de manera que las decisiones tomadas sean afines con los objetivos empresariales.

Luego, la gestión financiera al ser una técnica, puede evaluar la actuación operativamente de la empresa, además de ser factible poder realizar diagnósticos de situaciones actuales, así como efectuar predicciones sobre sucesos futuros. Además, se fundamenta en la medición del nivel de solvencia, liquidez y rentabilidad de la empresa.

Para Nava (2009), la gestión financiera engloba lo concerniente al dinero, a la inversión, administración y posesión del mismo, de manera que este sea manejado adecuadamente para que sea lucrativo; por ello su objetivo se centra en la maximización del valor de la inversión de los propietarios de la empresa. En definitiva, la gestión financiera de una empresa debe ser oportuna, confiable, $\mathrm{y}$, sobre todo, debe revisarse la capacidad de pago, para poder establecer el nivel de endeudamiento en el que se encuentra la empresa, para que logre la permanencia de su actividad productiva (Gitman y Zutter, 2016).

\section{Experiencias de empresas colombianas en crisis}

En las prácticas empresariales, los retos a los que se enfrenta la empresa colocan en prueba su permanencia en el mercado. Estos retos muestran la capacidad de la empresa para responder ante presiones como el incremento de competencia, alteraciones en la demanda, crecimientos acelerados, una economía cambiante, por citar sólo algunos. Ante estos retos, es importante identificar con rapidez las adversidades, para actuar antes de entrar en crisis, y realizar los cambios necesarios, porque su principal adversario es el tiempo.

Desde esta perspectiva, es fundamental que quienes manejan la empresa piensen y actúen más estratégicamente, que apelen a un pensamiento más crítico para realizar los análisis del entorno y tomar decisiones más sistémicas, es decir, que se piense y se tenga en cuenta a todos los que se involucren, así como los que puedan resultar impactados. Este apartado tiene como fin, dar a conocer los antecedentes y el contexto en el que se desarrollaron las ocho experiencias empresariales y describir las causales que las llevaron a la crisis (ver Cuadro 4), así como, las estrategias de eficiencia operacional y las condiciones que les permitieron un aprendizaje para el resurgimiento (las letras se refieren a las empresas que respondieron la encuesta). 


\section{Cuadro 4 \\ Causales de crisis en empresas colombianas}

\begin{tabular}{ll}
\hline \multicolumn{1}{c}{ Empresas } & \multicolumn{1}{c}{ Causales de Crisis } \\
\hline & $\begin{array}{l}\text { Financieros } \\
\text { Niveles de endeudamiento alto } \\
\text { No contar con flujo de caja } \\
\text { Baja liquidez }\end{array}$ \\
& $\begin{array}{l}\text { Deficiencias administrativas } \\
\text { F, b, g, h }\end{array}$ \\
& $\begin{array}{l}\text { Falta de preparación para la apertura } \\
\text { econica }\end{array}$ \\
f, h & Competencia desleal \\
& Competitividad \\
f & Sistema poco confiable y gobernable \\
c & Incumplimiento pago a proveedores \\
c & Productos no competitivos \\
\hline
\end{tabular}

Fuente: Elaboración propia, 2020.

Se coloca de manifiesto, que para la mayoría de las empresas objeto de este estudio, la gestión financiera fue evaluada por los directivos como una actuación operativa que incidió en la crisis y en los escenarios de incertidumbre económica, lo que las llevó a revisar sus estrategias e introducir cambios que respondieran al complejo proceso por el que estaban atravesando.

\subsection{Aprendizaje para el resurgimiento}

Los cambios generados para superar la crisis fueron catalogados por este estudio como "aprendizajes para el resurgimiento" porque, ante la complejidad y el caos la empresa se vuelve portadora del orden, de sentimientos, normas y confianza que son garantes de interacciones, así como premisas fundamentales del saber del empresario, características propias de un administrador.

Entre los aprendizajes para el resurgimiento empresarial, se evidencian percepciones y valoraciones como la creación de nuevos productos innovadores; la consolidación de las ventas nacionales e internacionales; el incremento y sostenibilidad en ventas; la oferta de bonos ordinarios en el mercado bursátil; el diseño de acuerdos con proveedores; la capitalización de pasivos financieros mediante bonos de riesgo; definir el target de mercado; innovar; calidad y mejoramiento continuo. Estos aprendizajes evidencian estrategias gerenciales para superar la crisis y sobrevivir en un entorno cada vez más dinámico y competitivo, que permitieron establecer distintos factores, como también variables orientadas al largo plazo y al desarrollo de las organizaciones empresariales.

Otro cambio que surge de las encuestas y que permitió reforzar la importancia del resurgimiento de las empresas fueron las "estrategias de eficiencia operacional", que son llevadas a cabo en todos los niveles organizacionales. Desarrollar capacidades para enfrentar la crisis y generar estrategias que reduzcan costos operacionales, los impactos y beneficios; para ello conviene realizar un estricto seguimiento de acciones y resultados.

Para los empresarios objeto de este estudio, existieron varias estrategias de eficiencia operación que les permitieron salir de la crisis, como: Introducir cambios para una gerencia más respetuosa y participativa; un código de buen gobierno; una reestructuración de las áreas organizacionales; generar estrategias publicitarias; desarrollar productos de calidad en los productos y servicios; innovar en sus marcas; construir una cultura organizacional, que genere confianza en el equipo; y, tener la voluntad para salir adelante después de la crisis. Las estrategias de eficiencia operacional fueron para las empresas un modelo de negocio, servicios, de redefinición, de iniciativas tácticas para racionalizar gastos y mostrar resultados a corto plazo, así como para maximizar el impacto a largo plazo (Porter, 2007).

Al realizar ajustes a los planes y prácticas organizacionales, para resurgir de la crisis y para gestionar estrategias de eficiencia operacional, se combinan múltiples factores que interactúan entre sí, proporcionando un mayor valor, siendo eficiente, lo que significa 
Olis Barreto, Irma Maria; Reyes, Giovanni E.; Martin-Fiorino, Victor y

Villalobos-Antúnez, José Vicente

Crisis empresarial, factores que influyen y alteran la gestión de las empresas en Colombia

desempeñarse en entornos de supervivencia o perdurabilidad empresarial.

\subsection{Impacto socioeconómico después de la crisis}

Se examina este tema, teniendo en cuenta la crisis empresarial por la que pasaron las empresas objeto de este estudio. Hay que reconocer y comprender las consecuencias, así como los efectos sociales y económicos que se vivieron. En ese contexto, la responsabilidad social de varias de las empresas tuvo que ver con el impacto y consecuencias sufridas por las personas que interactuaban con las empresas, talento humano, clientes, proveedores, entidades financieras, el Estado, dado que se alteró el trabajo, las relaciones, los valores, las ejecuciones y el reconocimiento de las empresas. Para el estudio, se identificaron aspectos socioeconómicos como el incremento en los empleos y el desarrollo social local. Algunas empresas mantuvieron la planta de personal por la experiencia que se tenía, en otros casos hubo despidos (Aguilera y Puerto, 2012).

Para los empresarios era importante tomar decisiones al menor coste social directo e indirecto, tanto para la propia empresa como para los colaboradores, proveedores, clientes, entidades financieras y el Estado. La experiencia de lucha de los empresarios, objeto de este estudio, permitieron un cambio en las empresas para que pudieran recuperarse. Fueron diversas las decisiones tomadas y autogestiones que debieron implementar, en su proceso de crisis. Se muestran las posibilidades de transformación, las tendencias y la relación con el entorno empresarial.

\section{Metodología}

Esta investigación buscó analizar los factores que intervienen y alteran el entorno de las empresas, en ese sentido, se pretende establecer las posibles tendencias y su relación con el entorno de las organizaciones. Con la descripción de las situaciones de crisis de las ocho empresas cuyos elementos en común se refieren al tipo de procesos de resurgimiento, las estrategias para su continuidad y el éxito de sus actuaciones para enfrentar las crisis en el contexto social colombiano, se busca comparar y constatar cómo estos factores pueden explicarse a partir de una realidad observada.

El diseño de la investigación fue de tipo no experimental, transversal, y descriptivo (Hernández, Fernández y Baptista, 2014), porque se observan los fenómenos tal y como ocurren naturalmente, no se interviene en su proceso; y se realiza una recolección de datos en un solo corte en el tiempo, a través de una encuesta y una entrevista semi-estructurada, aplicada a las empresas partícipes, con el propósito de estudiar varios factores que intervienen y perturban el entorno de las empresas.

Se planeó establecer las posibles tendencias y relaciones de los factores que permitieron a las empresas recuperarse de un estado de crisis. Se realizó la encuesta a 8 empresas, que pertenecen al sector comercial (3), al sector de servicios (2), y al sector manufacturero (3), por problemáticas en flujo de caja, deficiencias administrativas y de gobernabilidad, falta de preparación para la apertura económica y de competitividad.

Los criterios para la selección de las empresas fueron los siguientes: Empresas colombianas que han enfrentado crisis y han resurgido basado en los siguientes tres criterios fundamentales: 1) Recuperación del flujo de caja; 2) mayor dinamismo o rotación de los inventarios; $y, 3$ ) recuperación de la demanda efectiva en el mercado. Para efectos de esta investigación, se tomaron ocho empresas que se recuperaron de la crisis según los datos reflejados en las estadísticas de la Superintendencia Financiera de Colombia. En la Tabla 1, se identifican las empresas, sin referencia a sus datos específicos por necesarias razones de confidencialidad, y la edad de los 155 participantes que realizaron la encuesta. 
Tabla 1

Empresas, participantes y edades

\begin{tabular}{llccccccc}
\hline \multirow{2}{*}{ Edad } & $\mathbf{9}$ & $\mathbf{b}$ & $\mathbf{c}$ & $\mathbf{d}$ & $\mathbf{e}$ & $\mathbf{f}$ & $\mathbf{g}$ & $\mathbf{h}$ \\
\hline 18-31 Años & 1 & 19 & 2 & 3 & 1 & 6 & 7 & 2 \\
32-42 años & 2 & 9 & 14 & 4 & 0 & 13 & 8 & 13 \\
43-53 años & 2 & 7 & 9 & 1 & 1 & 9 & 4 & 7 \\
54-64 Años & 0 & 4 & 1 & 1 & 1 & 2 & 0 & 2 \\
> 65 Años & 0 & 1 & 0 & 0 & 0 & 1 & 1 & 0 \\
Totales & $\mathbf{5}$ & $\mathbf{4 0}$ & $\mathbf{2 6}$ & $\mathbf{9}$ & $\mathbf{3}$ & $\mathbf{3 1}$ & $\mathbf{2 0}$ & $\mathbf{2 4}$ \\
\hline
\end{tabular}

Fuente: Elaboración propia, 2020.

El estudio se apoyó en una entrevista semiestructurada y una encuesta, con información demográfica, nombre de la empresa, cargo del colaborador que respondió la encuesta, la empresa y la edad. En esta encuesta realizada a empresarios y personal administrativo de ocho empresas colombianas, se valoraban 47 items para los tres componentes de perdurabilidad: Gestión integral, gestión financiera, y eficiencia en los procesos descritos anteriormente. En el Cuadro 5, se relacionan los ítems (respuesta) que tiene cada componente: Como la encuesta es más genérica se tomaron solamente los items referidos a la perdurabilidad

\section{Cuadro 5}

\section{Componentes de perdurabilidad a analizar en la encuesta}

\begin{tabular}{cl}
\hline Componente & \multicolumn{1}{c}{ Ítems } \\
\hline & $\begin{array}{l}\text { La empresa eficiente es aquella que identifica, almacena, procesa, y utiliza la información } \\
\text { sobre la competencia, clientes y proveedores. (respuesta) }\end{array}$ \\
& $\begin{array}{l}\text { La forma como los socios y directivos gobiernan la empresa permite una operación eficiente. } \\
\text { (respuesta7) }\end{array}$ \\
$\begin{array}{c}\text { Eficiencia en los } \\
\text { procesos }\end{array}$ & $\begin{array}{l}\text { La correcta coordinación y eficiente comunicación entre los empleados depende del } \\
\text { conocimiento que tienen de sus responsabilidades y funciones, así como las del líder. } \\
\text { (respuesta8) }\end{array}$ \\
& $\begin{array}{l}\text { La empresa que cada día aprende de sus experiencias y las incorpora a su operación tiene } \\
\text { ventajas frente a sus competidores. (respuesta29) }\end{array}$ \\
Gestión Integral & $\begin{array}{l}\text { Es deseable el uso frecuente de la planeación estratégica en la construcción de futuro. } \\
\text { (respuesta15) }\end{array}$ \\
& $\begin{array}{l}\text { El buen gobierno depende de la capacidad de los directivos de tener en cuenta a sus } \\
\text { stakeholders (grupos de interés) en la toma de decisiones. (respuesta20) }\end{array}$ \\
& $\begin{array}{l}\text { La frecuente liquidación en empresas de un sector es producto de quiebra (pasivos totales } \\
\text { mayores que los activos), problemas de liquidez o ausencia de financiación a largo plazo. } \\
\text { (respuesta16) }\end{array}$
\end{tabular}

Fuente: Elaboración propia, 2020. 
Olis Barreto, Irma Maria; Reyes, Giovanni E.; Martin-Fiorino, Victor y

Villalobos-Antúnez, José Vicente

Crisis empresarial, factores que influyen y alteran la gestión de las empresas en Colombia

Para la valoración del cuestionario, se manejó una escala Likert, que servirá para aprehender la eficacia de las impresiones de los encuestados hacia los items planteados. Tiene cinco categorías de respuestas, donde 1 es "Totalmente en desacuerdo", y 5 "Totalmente de Acuerdo". El procedimiento utilizado para la recolección de los datos fue a través de encuestas, que se aplicaron directamente en las ocho empresas, mediante un link al cual podían acceder los participantes para resolverla. El análisis de los datos se realizó mediante SPSS.

\section{Resultados y discusión}

Los resultados de la investigación presentan, en una primera descripción, la propiedad psicométrica de las escalas de medida empleada. Se realizó un Análisis Factorial Exploratorio (AFE), que permitió agrupar los items (respuestas) que se correlacionan fuertemente entre sí, y cuyas relaciones con las variables de otros factores son menores (Pérez y Medrano, 2010). Con base en los anteriores supuestos, se realizó, inicialmente un AFE, en el que la prueba Kaiser Meyer Olkin (KMO) de adecuación de la muestra fue de 0.69 y la prueba de esfericidad de Bartlett mostró un valor crítico significativo de 0.00 .

Sin embargo, al analizar la matriz de componentes rotados, arroja que las variables que son significativas para el primer componente (eficiencia en los procesos), son las preguntas 8,15 y 20; y el segundo componente (gestión integral), con pesos superiores para las preguntas 4,7 y 29 . El promedio de la mayoría de las preguntas está por encima de 4 a diferencia de las preguntas 7 (Componente de Eficiencia en los procesos), y 16 (Gestión financiera). La puntuación más alta es para la pregunta 29 (La empresa que cada día aprende de sus experiencias y las incorpora a su operación tiene ventajas frente a sus competidores). Para el modelo no se incluye la respuesta 16 , debido a que no aparece significativo en ninguno de los componentes que arroja el análisis factorial.

Se volvió a realizar el AFE, excluyendo la respuesta 16 del componente de gestión financiera y los resultados fueron más favorables. Así como una vez analizada la Varianza total explicada (componentes de perdurabilidad) y el número de componentes, tal como aparecen en el primer renglón de la Tabla 2. Al respecto, los componentes referidos son: 1) Estabilidad del activo circulante; 2) compensación o reactivación del capital de trabajo; 3) amortiguamiento del pasivo circulante o deuda de corto plazo; 4) recuperación del flujo de caja; 5) rotación de inventario; y, 6) fortalecimiento del sector comercial; se observa que, en dos componentes de la escala ( 1 y 2 ), se explica el $54 \%$ de la perdurabilidad de la empresa.

Tabla 2

Varianza total explicada

\begin{tabular}{|c|c|c|c|c|c|c|c|c|c|}
\hline \multirow{2}{*}{ Componente } & \multicolumn{3}{|c|}{ Auto-valores iniciales } & \multicolumn{3}{|c|}{$\begin{array}{l}\text { Sumas de las saturaciones al } \\
\text { cuadrado de la extracción }\end{array}$} & \multicolumn{3}{|c|}{$\begin{array}{l}\text { Suma de las saturaciones al } \\
\text { cuadrado de la rotación }\end{array}$} \\
\hline & Total & $\begin{array}{c}\% \text { de la } \\
\text { varianza }\end{array}$ & $\begin{array}{c}\% \\
\text { acumulado }\end{array}$ & Total & $\begin{array}{c}\% \text { de la } \\
\text { varianza }\end{array}$ & $\begin{array}{c}\% \\
\text { acumulado }\end{array}$ & Total & $\begin{array}{c}\% \text { de } \\
\text { varianza }\end{array}$ & $\begin{array}{c}\% \\
\text { acumulado }\end{array}$ \\
\hline 1 & 2,156 & 35,935 & 35,935 & 2,156 & 35,935 & 35,935 & 1,778 & 29,627 & 29,627 \\
\hline 2 & 1,092 & 18,2 & 54,135 & 1,092 & 18,2 & 54,135 & 1,47 & 24,507 & 54,135 \\
\hline 3 & 0,865 & 14,415 & 68,549 & & & & & & \\
\hline 4 & 0,763 & 12,722 & 81,271 & & & & & & \\
\hline 5 & 0,632 & 10,541 & 91,813 & & & & & & \\
\hline 6 & 0,491 & 8,187 & 100 & & & & & & \\
\hline
\end{tabular}

Fuente: Elaboración propia, 2020. 
Luego, para identificar los pesos factores de los items, respecto de cada factor (Eficiencia en los procesos y gestión integral), se analizó la matriz de componentes rotados, evidenciando que los componentes antes mencionados están bien definidos (ver Tabla 3 ), dado que cumplen con las condiciones de contar con al menos tres items con pesos factoriales superiores a 0,50 (Morales, 2008).

Tabla 3

Matriz de componentes rotados (Varimax)

\begin{tabular}{lll}
\multicolumn{1}{c}{ Factor } & Componente & $\mathbf{2}$ \\
\hline Gestión Integral: Pregunta15 & 0,863 & \\
Gestión Integral: Pregunta20 & 0,644 & \\
Eficiencia en los procesos: Pregunta8 & 0,744 & \\
Eficiencia en los procesos: Pregunta4 & & 0,706 \\
Eficiencia en los procesos: Pregunta7 & & 0,758 \\
Eficiencia en los procesos: Pregunta29 & & 0,475 \\
\hline
\end{tabular}

Fuente: Elaboración propia (2020)

Se decidió analizar el comportamiento de los componentes con la comparación de medias por empresa. Se observan diferencias estadísticas significativas respecto de la variable gestión integral, entre las empresas objeto del estudio. En la variable eficiencia en los procesos, se observa diferencias estadísticas significativas entre dos de los items de las empresas analizadas (ver Tabla 4). 


\section{Tabla 4}

Anova Variables Eficacia en los procesos y Gestión integral

\begin{tabular}{|c|c|c|c|c|c|c|c|}
\hline & & & $\begin{array}{l}\text { Suma de } \\
\text { cuadrados }\end{array}$ & gl & $\begin{array}{l}\text { Media } \\
\text { cuadrática }\end{array}$ & $\mathbf{F}$ & Sig. \\
\hline \multirow{12}{*}{$\begin{array}{c}\text { Eficiencia } \\
\text { en los } \\
\text { procesos }\end{array}$} & Entre grupos & (Combinadas) & 7,285 & 7 & 1,041 & 1,036 & 0,409 \\
\hline & Dentro de grupos & & 147,709 & 147 & 1,005 & & \\
\hline & Total & & 154,994 & 154 & & & \\
\hline & Entre grupos & (Combinadas) & 5,026 & 7 & 0,718 & 0,803 & 0,586 \\
\hline & Dentro de grupos & & 131,451 & 147 & 0,894 & & \\
\hline & Total & & 136,477 & 154 & & & \\
\hline & Entre grupos & (Combinadas) & 4,6 & 7 & 0,657 & 0,844 & 0,553 \\
\hline & Dentro de grupos & & 114,471 & 147 & 0,779 & & \\
\hline & Total & & 119,071 & 154 & & & \\
\hline & Entre grupos & (Combinadas) & 1,36 & 7 & 0,194 & 0,518 & 0,82 \\
\hline & Dentro de grupos & & 55,182 & 147 & 0,375 & & \\
\hline & Total & & 56,542 & 154 & & & \\
\hline \multirow{6}{*}{$\begin{array}{l}\text { Gestión } \\
\text { Integral }\end{array}$} & Entre grupos & (Combinadas) & 5,26 & 7 & 0,751 & 1,208 & 0,302 \\
\hline & Dentro de grupos & & 91,411 & 147 & 0,622 & & \\
\hline & Total & & 96,671 & 154 & & & \\
\hline & Entre grupos & (Combinadas) & 6,054 & 7 & 0,865 & 1,782 & 0,095 \\
\hline & Dentro de grupos & & 71,339 & 147 & 0,485 & & \\
\hline & Total & & 77,394 & 154 & & & \\
\hline
\end{tabular}

Fuente: Elaboración propia, 2020.

Al analizar de forma más particularizada, en cada una de las variables no se observan diferencias significativas entre las medias por empresa y por tipo de cargo (ver Tabla 5).
En cuanto, al tipo de cargo de las personas que contestan no se observan diferencias significativas entre los promedios. 
Tabla 5

Anova Variables por empresa y por tipo de cargo

\begin{tabular}{|c|c|c|c|c|c|c|c|}
\hline & & & $\begin{array}{l}\text { Suma de } \\
\text { cuadra- } \\
\text { dos }\end{array}$ & gl & $\begin{array}{l}\text { Media } \\
\text { cuadrática }\end{array}$ & $\mathbf{F}$ & Sig. \\
\hline \multirow{12}{*}{$\begin{array}{c}\text { Eficiencia en los } \\
\text { procesos }\end{array}$} & Entre grupos & (Combinadas) & 7,285 & 7 & 1,041 & 1,036 & 0,409 \\
\hline & Dentro de grupos & & 147,709 & 147 & 1,005 & & \\
\hline & Total & & 154,994 & 154 & & & \\
\hline & Entre grupos & (Combinadas) & 5,026 & 7 & 0,718 & 0,803 & 0,586 \\
\hline & Dentro de grupos & & 131,451 & 147 & 0,894 & & \\
\hline & Total & & 136,477 & 154 & & & \\
\hline & Entre grupos & (Combinadas) & 4,6 & 7 & 0,657 & 0,844 & 0,553 \\
\hline & Dentro de grupos & & 114,471 & 147 & 0,779 & & \\
\hline & Total & & 119,071 & 154 & & & \\
\hline & Entre grupos & (Combinadas) & 1,36 & 7 & 0,194 & 0,518 & 0,82 \\
\hline & Dentro de grupos & & 55,182 & 147 & 0,375 & & \\
\hline & Total & & 56,542 & 154 & & & \\
\hline \multirow{6}{*}{$\begin{array}{l}\text { Gestión Inte- } \\
\text { gral }\end{array}$} & Entre grupos & (Combinadas) & 5,26 & 7 & 0,751 & 1,208 & 0,302 \\
\hline & Dentro de grupos & & 91,411 & 147 & 0,622 & & \\
\hline & Total & & 96,671 & 154 & & & \\
\hline & Entre grupos & (Combinadas) & 6,054 & 7 & 0,865 & 1,782 & 0,095 \\
\hline & Dentro de grupos & & 71,339 & 147 & 0,485 & & \\
\hline & Total & & 77,394 & 154 & & & \\
\hline
\end{tabular}

Fuente: Elaboración propia, 2020.

\section{Conclusiones}

En momentos de crisis hay que estudiar y analizar detenidamente los principios de la empresa más que nunca, volver a la razón de ser de la empresa para no perder el norte. Las crisis empresariales despiertan muchas expectativas, porque generalmente se encuentran sin recursos, sin escasez o con restricciones; por ende, las prerrogativas son otras, las políticas comunes de seguridad o riesgo que le permitan a la empresa evaluar permanentemente su entorno general y específico.

Al convivir y relacionarse la empresa con su entorno, genera crecimientos constantes que determinaran su posicionamiento $\mathrm{y}$ perdurabilidad en el mercado. Pero también, deja aprendizajes que implican hacer un examen de las lecciones aprendidas en sus propias experiencias, y por qué no, en la de las experiencias de otras organizaciones. En una crisis empresarial, el avance real no es fomentar el retraimiento, los temores o tradicionalismos, es más bien ceder en algunos procesos de construcción encaminados a ideas de futuro, porque en un mundo tan dinámico y competitivo, las decisiones que se toman a tiempo son la salida más sabia.

Como resultado de este estudio, se evidencia que, para potenciar las soluciones a las crisis, las operaciones de las empresas deben impulsar el espíritu de sus 
Olis Barreto, Irma Maria; Reyes, Giovanni E.; Martin-Fiorino, Victor y

Villalobos-Antúnez, José Vicente

Crisis empresarial, factores que influyen y alteran la gestión de las empresas en Colombia

operaciones, lo cual debe ser el resultado de una respuesta colectiva y solidaria de todos los participantes en la organización. Por otra parte, los resultados de los análisis colocan de manifiesto la necesidad de evitar que se pueda llegar a un punto de crisis de no retorno, por la no eficiencia en los procesos o en la gestión integral, que no permita recuperar la empresa.

Las empresas cuentan generalmente con capacidades de defensa propia, no dependen de agentes externos incontrolables, por eso es preciso desplegar visibilidad, utilidad y cultura, que se asocie con las necesidades del entorno, en un todo ineludible, suplementario y esencial, que le permita afrontar riesgos o amenazas. Indudablemente, hay que redimensionar las capacidades básicas de la empresa, proteger las iniciativas, garantizando el futuro, aportarle al interés empresarial, a las estrategias, a las capacidades del equipo de trabajo, porque no es un proceso aislado, es integral.

El artículo aporta una visión de los elementos centrales del choque simultáneo de oferta y demanda que afectó a una gran mayoría de las empresas en el entorno colombiano, provocando efectos negativos especialmente en los sectores más vulnerables. A partir de los resultados del estudio, es posible concluir que son diversos los factores que intervienen y afectan el entorno de las empresas en Colombia, tomando en cuenta que cada empresa cuenta con particularidades específicas heterogéneas. En cuanto a los componentes analizados, relativos a la eficiencia en los procesos, gestión financiera y gestión integral, la revisión literaria evidencia la importancia que tiene prever estos factores y lo que se alcanza al predecir la crisis empresarial.

Finalmente, existe la posibilidad de investigaciones futuras que, desde una perspectiva interdisciplinaria para el abordaje de las crisis empresariales, profundicen y abran espacios para reflexionar sobre los comportamientos recurrentes del entorno empresarial, las tendencias y relaciones con el entorno.

\section{Referencias bibliográficas}

Aguilera, A., y Puerto, D. P. (2012). Crecimiento empresarial basado en la Responsabilidad Social. Pensamiento \& Gestión, (32), 1-26.

Agulló, C (1999). Cambios significativos en el mundo empresarial. Economía Industrial, (330), 11-18.

Antonacopoulou, E. P., y Sheaffer, Z. (2014). Learning in crisis: Rethinking the relationship between organizational learning and crisis management. Journal of Management Inquiry, 23(1), 5-21. https://doi. org/10.1177/1056492612472730

Báez, M. D. P., y Puentes, G. A. (2018). Parámetros financieros para la toma de decisiones en pequeñas y medianas empresas del municipio Duitama, Colombia. Revista de Ciencias Sociales (Ve), XXIV(1), 67-84.

Barney, J. B. (2010). Gaining and sustaining competitive advantage. Pearson.

Blanchard, O., Dell'Ariccia, G., y Mauro, P. (2010). Rethinking macroeconomic policy. Journal of Money, Credit and Banking, 42(s1), 199-215.

Blanco-Ariza, A. B., Vásquez-García, Á. W., García-Jiménez, R., y MelamedVarela, E. (2020). Estructura organizacional como determinante competitivo en pequeñas y medianas empresas del sector alimentos. Revista de Ciencias Sociales (Ve), XXVI(2), 133-147. https://doi.org/10.31876/rcs. $\underline{\mathrm{v} 26 \mathrm{i} 2.32429}$

Bom-Camargo, Y. I. (2021). Hacia la responsabilidad social como estrategia de sostenibilidad en la gestión empresarial. Revista de Ciencias Sociales (Ve), XXVII(2), 130-146. https://doi.org/10.31876/rcs. v27i2.35903 
Castro, Y. (2007). La influencia del entorno en el desempeño de las organizaciones. Particularidades del entorno empresarial cubano. Economía y Desarrollo, 142(2), 136-149.

Collins, J. C., y Porras, J. I. (2006). Empresas que perduran: Principios exitosos de compañias triunfadoras. Grupo Editorial Norma.

Deverell, E., y Olsson, E-K. (2010). Organizational culture effects on strategy and adaptability in crisis management. Risk Management, 12(2), 116-134.

Gitman, L. J., y Zutter, C. J. (2016). Principios de Administración financiera. Pearson Educación.

González, A. (2008). Marketing preventivo: La comunicación de crisis en la empresa. S.A. Bosch.

Grant, R. M. (2005). Contemporary strategy analysis: Concepts, techniques, applications. Blackwell Publishing.

Hannan, M. T., y Freeman, J. (1977). The population ecology of organizations. American Journal of Sociology, 82(5), 929-964.

Hernández, R., Fernández, C., y Baptista, M. D. P. (2014). Metodología de la investigación. McGraw-Hill/ Interamericana Editores.

Jaques, T. (2010). Reshaping crisis management: The challenge for organizational design. Organization Development Journal, 28(1), 9-17.

Kast, F. E., y Rosenzweig, J. E (1972). General systems theory: Applications for organization and management. Academy of Management Journal, 15(4), 447-465. https://doi. org/10.5465/255141

Koontz, H., Weihrich, H., y Cannice, M. (2012). Administración. Una perspectiva global y empresarial. McGraw-Hill/
Interamericana Editores.

Levy, M., y Hadar, I. (2010). Teaching MBA students the use of Web2.0: The knowledge management perspective. Journal of Information Systems Education, 21(1), 55-67.

Maldonado, C. E. (Ed.) (2009). Complejidad: Revolución científica y teoría. Editorial Universidad del Rosario.

Márquez, L. E., Viteri, M. J., Useche, L. M., y Cuétara, L. M. (2021). Proceso administrativo y sostenibilidad empresarial del sector hotelero de la parroquia Crucita, Manabí-Ecuador. Revista de Ciencias Sociales (Ve), XXVII(2), 367-385. https://doi. org/10.31876/rcs.v27i2.35931

Martín, N., Hernangómez, J., y Martín, V. (2007). El deleite de la eficiencia. UCJC Business and Society Review, 2(14), 56-67.

Martínez, H. R., Cazallo, A. M., Meñaca, I., y Uribe, C. M. (2020). Desempeño financiero de las empresas minoristas de alimentos y bebidas en Barranquilla - Colombia. Revista de Ciencias Sociales (Ve), XXVI(1), 144160. https://doi.org/10.31876/rcs. $\underline{\mathrm{v} 26 \mathrm{i} 1.31316}$

McCray, J. P., Gonzalez, J. J., y Darling, J. $R$ (2012). Transformational crisis management in organizational development: A focus on the case of Barnes \& Noble vs. Amazon. Organization Development Journal, 30(1), 39-52.

Mintzberg, H. (2005). La estructuración de las organizaciones. Editorial Ariel S. A.

Morales, P. (2008). Estadística aplicada a las Ciencias Sociales: La fiabilidad de los test y escalas. Universidad Pontificia Comillas.

Nava, M. A. (2009). Análisis financiero: Una herramienta clave para una 
Olis Barreto, Irma Maria; Reyes, Giovanni E.; Martin-Fiorino, Victor y

Villalobos-Antúnez, José Vicente

Crisis empresarial, factores que influyen y alteran la gestión de las empresas en Colombia

gestión financiera eficiente. Revista Venezolana de Gerencia, 14(48), 606628

Pearson, C. M., y Clair, J. A (1998). Reframing crisis management. The Academy of Management Review, 23(1), 59-76. https://doi.org/10.2307/259099

Pearson, C. M., y Mitroff, I. I. (1993). From crisis prone to crisis prepared: A framework for crisis management. The Executive, 7(1), 48-59.

Pérez, E., y Medrano, L. (2010). Análisis factorial exploratorio: Bases conceptuales y metodológicas. Revista Argentina de Ciencias del Comportamiento, 2(1), 58-66.

Pérez, G. (2002). Investigación cualitativa. Retos e interrogantes. La Muralla.

Porter, M. E. (2001). Estrategia competitiva. Técnicas para el análisis de los sectores industriales y de la competencia. Compañia Editorial Continental.

Porter, M. E (2007). La ventaja competitiva de las naciones. Harvard Business Review, 85(11), 69-95.

Real Academia Española (2021). Diccionario de la lengua española. Eficiencia. https://dle.rae.es/eficiencia

Restrepo, L. F., Vélez, A. R., Méndez, C. E., Rivera-Rodríguez, H-A., y Mendoza, L. A. (2009). Aproximación a una metodología para la identificación de componentes que crean condiciones para la perdurabilidad en empresas colombianas. Documento de Investigación (39). Editorial Universidad del Rosario.

Rivera, H. A. (2012). Perdurabilidad empresarial: Concepto, estudios, hallazgos. Cuadernos de Administración, 28(47), 105-115.

Rivera, H. A., y Cardona, D (2012). Protocolo de investigación. Working Paper
(145). Editorial Universidad del Rosario. $\quad$ http://dx.doi.org/10.2139/ $\underline{\text { ssrn. } 2275417}$

Sansores, E. A., Navarrete, J. E., AlvaradoPeña, L. J., y Licandro, Ó. D. (2020). Diagnóstico situacional en microempresas mexicanas: Fracaso o sobrevivencia empresarial. Revista de Ciencias Sociales (Ve), XXVI(1), 61-76. https://doi.org/10.31876/rcs. $\underline{\mathrm{v} 26 \mathrm{i} 1.31311}$

Toca, C. E (2011). Perspectivas para el estudio de la realidad y la perdurabilidad de las organizaciones. Documento de Investigación (98). Editorial Universidad del Rosario.

Tommasetti, A., y Martin, V. (2013). De la eficacia al sentido. Ética, economía y empresa en los debates contemporáneos. Cultura Latinoamericana, 2(18), 99-122.

Valero, J. M. (2012). Programa Barrio Adentro I: Una estrategia de gestión pública en el estado Zulia. Revista Venezolana de Gerencia, 17(59), 529-547.

Veil, S. R. (1973). Mindful learning in crisis management. Journal of Business Communication, 48(2), 116-147. $\quad$ https://doi. org/10.1177/0021943610382294

Vélez, A. R. (2005). Aprendizajes estratégicos para la perdurabilidad: reseña de investigación de casos latinoamericanos. Universidad Empresa, 7(9), 86-99.

Westphalen, M. H., y Piñuel, J. L. (1993). La dirección de comunicación. Del Prado Ediciones. 